Pacific Journal of Mathematic 


\title{
REPRODUCING KERNELS IN SEPARABLE HILBERT SPACES
}

\section{HAYRI KÖREZLIOĞLU}

\begin{abstract}
A theorem on the existence of a reproducing kernel in a separable Hilbert space of functions is proved. As an application of this theorem, a method of interpolation of the functions in a separable Hilbert space with a reproducing kernel is given. This method is used to construct the elements of the Hilbert space generated by a second order stochastic process, in case this space is separable.
\end{abstract}

Theorems 2,3 and 4 of this paper, which were motivated by Parzen's work [2], [3], were originally proved in somewhat different form in collaboration with J. Ricatte [4]. In this paper it will be shown that these three theorems are the consequences of a more general statement given in what follows as Theorem 1.

1. Preliminaries. Let $\mathfrak{S}$ be a Hilbert space of real or complex functions defined on an arbitrary set $T$. The scalar product of any ordered pair of functions $f, g$ in $\mathfrak{F}$ will be denoted by $\langle f, g\rangle$ and the norm of a function $f \in \mathfrak{S}$ by $\|f\|$. A two variable function $K$ defined on the product set $T \times T=T^{2}$ is the reproducing kernel of $\mathfrak{Z}$, if it satisfies the following two conditions:

(A) $K(t, \cdot) \in \mathfrak{S}, \forall t \in T$.

(B) $\langle f, K(t, \cdot)\rangle=f(t), \forall t \in T$ and $\forall f \in \mathfrak{W}$.

The last property is called reproduction property of $K^{1}$.

$K$ is self-reproducing, i.e. $K(t, \tau)=\langle K(t, \cdot), K(\tau, \cdot)\rangle$. It is positive-semi-definite, i.e.

$$
\sum_{i, j \in I} \lambda_{i} \bar{\lambda}_{j} K\left(t_{i}, t_{j}\right)=\left\|\sum_{i \in I} K\left(t_{i}, \cdot\right)\right\|^{2}>0, \lambda_{i} \in C, \forall_{i} \in I \subset N .
$$

(where $C$ is the set of complex numbers, $I$ an arbitrary finite subset of the set $N$ of positive integers and $\bar{\lambda}_{j}$ the conjugate of $\lambda_{j}$ ). In particular, $K$ has the Hermitian symmetry $(K(t, \tau)=\bar{K}(\tau, t), \forall t, \tau \in T)$ and

$$
0 \leqq\|K(t, \cdot)\|^{2}=K(t, t)<\infty, \forall t \in T .
$$

If $\mathfrak{S}$ has a reproducing kernel, this kernel is always unique, for if $K$ and $K^{\prime}$ were two distinct reproducing kernels of $\mathfrak{S}$, their reproduction property would imply

${ }^{1}$ For a more general and detailed presentation of the Theory of Reproducing Kernels, see the article by Aronzajn [1]. 


$$
K(t, \tau)=\left\langle K(t, \cdot), K^{\prime}(\tau, \cdot)\right\rangle=\left\langle\overline{K^{\prime}(\tau, \cdot), K(t, \cdot)}\right\rangle=\bar{K}^{\prime}(\tau, t)=K^{\prime}(t, \tau) .
$$

The weak convergence (consequently the strong convergence) of a sequence $\left\{f_{n}\right\} \subset \mathfrak{S}$ to a function $f \in \mathfrak{S}$ implies its pointwise convergence to the same function $f$, for

$$
\lim _{n \rightarrow \infty} f_{n}(t)=\lim _{n \rightarrow \infty}\left\langle f_{n}, K(t, \cdot)\right\rangle=\langle f, K(t, \cdot)\rangle=f(t) .
$$

If a topology is defined on $T$, then the continuity of $K$ with respect to the product topology on $T^{2}$ implies the continuity of each function in $\mathfrak{S}$. This is the consequence of the Schwarz inequality applied to $(B)$ :

$$
\begin{aligned}
\left|f(t)-f\left(t_{0}\right)\right|^{2} & =\left|\left\langle f, K(t, \cdot)-K\left(t_{0}, \cdot\right)\right\rangle\right|^{2} \\
& \leqq\|f\|^{2}\left[K(t, t)-K\left(t, t_{0}\right)-K\left(t_{0}, t\right)+K\left(t_{0}, t_{0}\right)\right] .
\end{aligned}
$$

Given a finite and positive-semi-definite function $K$ on $T^{2}$, there exists a uniquely defined Hilbert space of functions on $T$, whose reproducing kernel is $K$ (Moore's Theorem). This space is obtained in the following way: Let $L_{K}$ be the linear set generated by $\{K(t, \cdot)$, $t \in T$,$\} i.e. the set of all finite linear combinations$

$$
\sum_{i} \lambda_{i} K\left(t_{i}, \cdot\right), \lambda_{i} \in C,
$$

Let a scalar product of any ordered pair of elements $f, g \in L_{K}$ be defined by

$$
\langle f, g\rangle=\sum_{i, j} \lambda_{i} \bar{\mu}_{j} K\left(t_{i}, t_{j}\right)
$$

where

$$
f=\sum_{i} \lambda_{i} K\left(t_{i}, \cdot\right), g=\sum_{j} \mu_{j} K\left(t_{j}, \cdot\right) .
$$

This scalar product induces a norm on $L_{K}$, so that $L_{K}$ is a preHilbert space. Obviously

$$
f(t)=\langle f, K(t, \cdot)\rangle, \forall t \in T \text { and } \forall f \in L_{K} .
$$

If $\left\{f_{n}\right\}$ is a Cauchy sequence in $L_{K}$, then $\left\{f_{n}\right\}$ converges everywhere to a function $f$, for

$$
\left|f_{m}(t)-f_{n}(t)\right|^{2} \leqq\left\|f_{m}-f_{n}\right\|^{2} K(t, t) .
$$

If the norm of $f$ is defined by $\|f\|=\lim _{n \rightarrow \infty}\left\|f_{n}\right\|$, the space obtained by the adjunction to $L_{K}$ of pointwise limits of Cauchy sequences in $L_{K}$ is a Hilbert space and $K$ reproduces all functions of this space. The space generated by $\{K(t, \cdot), t \in T\}$ will be denoted by $\mathfrak{S}_{K}$.

Let $\mathfrak{S}$ be any Hilbert space whose reproducing kernel is $K$. Then 
the class $\{K(t, \cdot), t \in T\}$ is a basis for $\mathfrak{S}_{2}$, so that $\mathfrak{g}$ coincides with $\mathfrak{L}_{K}$. Consequently, if a closed subspace $\mathfrak{S}$ of a Hilbert space $\mathfrak{h}$ of functions on $T$ has a reproducing kernel $K$, then for any function $h \in \mathfrak{h}$, the scalar product $\langle h, K(t, \cdot)\rangle$ gives the projection of $h$ onto $\mathfrak{F}$. Also, if $\mathscr{L}$ is a closed subspace of $\mathfrak{S}_{K}$, then the reproducing kernel of $\mathscr{L}$ is the projection $\hat{K}(t, \cdot)$ of $K(t, \cdot)$ onto $\mathscr{L}$.

2. The case of separable Hilbert spaces. The following theorem gives a necessary and sufficient condition for a separable Hilbert space of functions to have a reproducing kernel.

THEOREM 1. Let $\mathfrak{S}$ be a separable Hilbert space of functions defined on $T$ and let $\left\{e_{i}\right\}$ be a countable class of linearly independent functions in $\mathfrak{S}$ forming a basis for $\mathfrak{S}$. Let $\left\{K_{n}\right\}$ be the sequence defined by

$$
K_{n}(t, \tau)=\sum_{i, j=1}^{n} \bar{e}_{i}(t) \gamma_{i j n} e_{j}(\tau)
$$

where $\left(\gamma_{i j n}\right)_{1 \leqq i, j \leqq n}$ is the inverse of the matrix $\left(\left\langle e_{i}, e_{j}\right\rangle\right)_{1 \leqq i, j \leqq n}$.

$\left(C_{1}\right)$ If $\forall t \in T,\left\{K_{n}(t, t)\right\}$ converges as $n \rightarrow \infty$, then any Cauchy sequence $\left\{\sum_{i=1}^{n} \alpha_{n, i} e_{i}\right\} \subset \mathfrak{S}$ converges everywhere on $T$.

$\left(C_{2}\right)$ If, moreover, pointwise limits of such Cauchy sequences coincide with their limits in norm,

then $K(t, \tau)=\lim _{n \rightarrow \infty} K_{n}(t, \tau)$, which exists $\forall t, \tau \in T$, is the reproducing kernel of $\mathfrak{S}$.

Conversely, if $\$$ has a reproducing kernel $K$, then the conditions $C_{1}$ and $C_{2}$ are fulfilled and $\forall t, \tau \in T, K(t, \tau)=\lim _{n \rightarrow \infty} K_{n}(t, \tau)$.

Proof. To avoid all trivialities, $\mathfrak{S}$ can be supposed to be infinite dimensional.

Sufficiency of $C_{1}$ and $C_{2}$. Consequences of $C_{1}$. Let $\mathfrak{g}_{n}$ be the subspace generated by $\left\{e_{i}, 1 \leqq i \leqq n\right\} . \quad K_{n}(t, \cdot)$ is obviously an element of $\mathfrak{S}_{n}$ and it reproduces all functions in $\mathfrak{S}_{2}$. Moreover, $\mathfrak{L}_{n} \subset \mathfrak{S}_{m}$ for $m>n$. Then $K_{n}(t, \cdot)$ is the projection of $K_{m}(t, \cdot)$ onto $\mathfrak{Q}_{n}$. Consequently, the relations

$$
\begin{aligned}
\left\langle K_{m}(t, \cdot), K_{n}(\tau, \cdot)\right\rangle & =K_{n}(t, \tau), m>n, \\
\left\|K_{m}(t, \cdot)-K_{n}(t, \cdot)\right\|^{2} & =K_{m}(t, t)-K_{n}(t, t), m>n,
\end{aligned}
$$

hold. By the last relation, it can be seen that $\left\{K_{n}(t, t)\right\}$ is an increasing sequence which converges by hypothesis, so that $\left\{K_{n}(t, \cdot)\right\}$ is a Cauchy 
sequence in $\mathfrak{S}$ for every $t \in T$. Let $K(t, \cdot)$ be the limit of this sequence. For a given function $f \in \mathfrak{S}$, the function $f_{n}$ defined by

$$
\hat{f}_{n}(t)=\left\langle f, K_{n}(t, \cdot)\right\rangle=\sum_{i, j=1}^{n} \beta_{i} \gamma_{i j n} e_{j}(t), \beta_{i}=\left\langle f, e_{i}\right\rangle
$$

is the projection of $f$ onto $\mathfrak{Q}_{n}$. Thus, the relations

$$
\begin{gathered}
\left\|f-\hat{f}_{n}\right\|^{2}=\left\|f_{n}\right\|^{2}-\left\|\hat{f}_{n}\right\|^{2} \\
\left\|\hat{f}_{m}-\hat{f}_{n}\right\|^{2}=\left\|\hat{f}_{m}\right\|^{2}-\left\|\hat{f}_{n}\right\|^{2}, \quad m>n \\
\left\|\hat{f}_{n}\right\| \leqq\left\|\hat{f}_{m}\right\| \leqq\|f\|, \quad m>n .
\end{gathered}
$$

hold. Consequently, $\{\|\hat{f}\|\}$ is a nondecreasing sequence bounded by $\|f\|$, therefore it converges. Then, according to (6), $\left\{f_{n}\right\}$ is a Cauchy sequence in $\mathfrak{S}$.

Let us suppose that

$$
f_{n}=\sum_{k=1}^{n} \alpha_{n, i} e_{i}
$$

is a sequence converging to $f$. Since $f_{n} \in \mathfrak{S}_{n}$, the relation

$$
\left\langle f, f_{n}\right\rangle=\left\langle f-\hat{f}_{n}+\hat{f}_{n}, f_{n}\right\rangle=\left\langle\hat{f}_{n}, f_{n}\right\rangle
$$

holds. Then, $\lim _{n \rightarrow \infty}\left\langle\hat{f}_{n}, f_{n}\right\rangle=\lim _{n \rightarrow \infty}\left\langle f, f_{n}\right\rangle=\|f\|^{2}$, and according to (7),

$$
\begin{aligned}
0 & \leqq \lim _{n \rightarrow \infty}\left\|\hat{f}_{n}-f_{n}\right\|^{2}=\lim _{n \rightarrow \infty}\left(\left\|\hat{f}_{n}\right\|^{2}-\left\langle\hat{f}_{n}, f_{n}\right\rangle-\left\langle f_{n}, \hat{f}_{n}\right\rangle+\left\|f_{n}\right\|^{2}\right) \\
& =\lim _{n \rightarrow \infty}\left\|\hat{f}_{n}\right\|^{2}-\|f\|^{2} \leqq 0 .
\end{aligned}
$$

Consequently, $\lim _{n \rightarrow \infty}\left\|\hat{f}_{n}\right\|=\|f\|$. Then the relation (5) shows that $\left\{\hat{f}_{n}\right\}$ converges to $f$ in norm.

Since the strong convergence of $\left\{\hat{f}_{n}\right\}$ implies its weak convergence, one has

$$
\begin{aligned}
\lim _{n \rightarrow \infty} \hat{f}_{n}(t) & =\lim _{n \rightarrow \infty}\left\langle\hat{f}_{n}, K(t, \cdot)\right\rangle \\
& =\langle f, K(t, \cdot)\rangle=g(t) .
\end{aligned}
$$

Thus, $\left\{\hat{f}_{n}\right\}$ converges everywhere. From this, it is easy to see that any Cauchy sequence of the type (8) also converges everywhere. In fact,

$$
\hat{f}_{n}(t)-f_{n}(t)=\left\langle f-f_{n}, K_{n}(t, \cdot)\right\rangle .
$$

By applying the Schwarz inequality and taking into account the fact 
that $K_{n}(t, t)<K(t, t)$, one can write

$$
\left|\hat{f}_{n}(t)-f_{n}(t)\right|^{2} \leqq\left\|f-f_{n}\right\|^{2} K_{n}(t, t) \leqq\left\|f-f_{n}\right\|^{2} K(t, t) .
$$

Since $\left\{f_{n}\right\}$ converges to $f$ in norm, it is seen that $\lim _{n \rightarrow \infty}\left|\hat{f}_{n}(t)-f_{n}(t)\right|={ }_{0}^{0}$. Finally, the inequality

$$
\left|g(t)-f_{n}(t)\right| \leqq\left|g(t)-\hat{f}_{n}(t)\right|+\left|\hat{f}_{n}(t)-f_{n}(t)\right|
$$

shows that $\left\{f_{n}(t)\right\}$ converges to the same limit $g(t)$ as $\left\{\hat{f}_{n}(t)\right\}$.

Consequences of $C_{2}$. In case the pointwise limit and the limit in norm of Cauchy sequences of the type (8) coincide, then by (9) the reproduction property $g(t)=f(t)=\langle f, K(t, \cdot)\rangle$ is obtained. Also, the sequence $\left\{K_{n}(t, \tau)\right\}$ converges to $K(t, \tau), \forall t, \tau \in T$. Hence, $K(t, \tau)=$ $\lim _{n \rightarrow \infty} K_{n}(t, \tau)$ is the reproducing kernel of $\mathfrak{g}$.

Necessity of $C_{1}$ and $C_{2}$. Suppose that $\mathfrak{S}$ possesses a reproducing kernel $K$. The relation (3) which is still valid, together with the relation

$$
\left\|K(t, \cdot)-K_{n}(t, \cdot)\right\|^{2}=K(t, t)-K_{n}(t, t),
$$

obtained from (5) by replacing $f(\cdot)$ by $K(t, \cdot)$, imply that

$$
K_{n}(t, t)<K_{m}(t, t)<K(t, t) \quad \text { for } m>n \text {. }
$$

Thus, $\left\{K_{n}(t, t)\right\}$ is an increasing sequence bounded by $K(t, t)<\infty$. Hence, it converges, so that the condition $C_{1}$ is fulfilled. On the other hand, since $\mathfrak{S}$ possesses a reproducing kernel, the condition $C_{2}$ is automatically fulfilled.

Consequently, $\lim _{n \rightarrow \infty} K_{n}(t, \tau)$ is a reproducing kernel of $\mathfrak{S}$. Reproducing kernel being always unique, one has $K(t, \tau)=\lim _{n \rightarrow \infty} K_{n}(t, \tau)$.

REMARK. If only the condition $C_{1}$ holds, then the space $\mathfrak{S}$ can be made isomorphic to a Hilbert space whose reproducing kernel is $\Gamma(t, \tau)=\langle K(t, \cdot), K(\tau, \cdot)\rangle$ with $K(t, \cdot)$ as the strong limit of $\left\{K_{n}(t, \cdot)\right\}$ in $\mathfrak{S}$. In fact, any Cauchy sequence of the type (8) converging to $f \in \mathfrak{S}$ converges everywhere in $T$ to a function $g$. As in the theorem of Moore, if the set of all linear combinations of the functions $\left\{e_{i}\right\}$ is completed by the adjunction of pointwise limits of Cauchy sequences of this set with respect to the topology of $\mathfrak{S}$, and if the limit of the norms for each sequence is assigned as the norm of the pointwise limit of the sequence, then a Hilbert space $\mathfrak{S}_{\Gamma}$ is obtained. The reproducing kernel of $\mathfrak{S}_{\Gamma}$ turns out to be $\Gamma$. This latter space is obviously isomorphic to $\mathfrak{S}$. This isomorphism can be represented by 


$$
g(t)=\langle f, K(t, \cdot)\rangle, \forall t \in T, f \in \mathfrak{S} \text { and } g \in \mathfrak{S}_{\Gamma} \cdot
$$

It can be proved also, that the class of functions $\{K(t, \cdot), t \in T\}$ generates $\mathfrak{S}$, in the sense that it is a basis for $\mathfrak{S}$, that is, any function $f \in \mathfrak{g}$ for which $\langle f, K(t, \cdot)\rangle=0$ for all $t \in T$, has its norm equal to zero. In fact, let $f$ be such a function. Then the function $g \in \mathfrak{S}_{\Gamma}$ corresponding to $f$ in the isomorphism between $\mathfrak{S}_{2}$ and $\mathfrak{S}_{\Gamma}$ is the null function in $\mathfrak{S}_{\Gamma}$. Consequently, its norm and the norm of $f$ equal zero.

It is worth mentionning that in view of this remark and the following theorem, there exists a countable subset $S$ of $T$ such that $K=\Gamma$ on both $S \times T$ and $T \times S$.

In what follows, a separable Hilbert space $\mathfrak{S}_{K}$ of functions on $T$, with reproducing kernel $K$, will be considered. Since the class $\{K(t, \cdot), t \in T\}$ generates $\mathfrak{L}_{K}$, there exists a countable subset $S$ of $T$ such that $\left\{K\left(t_{i}, \cdot\right), t_{i} \in S, i \in N\right\}$ is a class of linearly independent functions forming a basis for $\mathfrak{S}_{K}$. The matrix $\left(\gamma_{i j n}\right)_{1 \leqq i, j \leqq n}$ will denote the inverse of the matrix $\left(K\left(t_{i}, t_{j}\right)\right)_{1 \leqq i, j \leqq n}$ and $S_{n}$ will denote $\left\{t_{1}, t_{2}, \cdots, t_{n}\right\} \subset S$.

THEOREM 2. For any function $f \in \mathfrak{S}_{K}$, the sequence of functions defined by

$$
\widehat{f}_{n}(\cdot)=\sum_{i, j=1}^{n} f\left(t_{i}\right) \gamma_{i j n} K\left(t_{j}, \cdot\right)
$$

converges to $f$, as $n \rightarrow \infty$, (both in norm and everywhere).

Proof. To prove the theorem, it suffices to replace $e_{i}$ by $K\left(t_{i}, \cdot\right)$ in the preceding theorem. Then $K_{n}(t, \tau)$ becomes

$$
K_{n}(t, \tau)=\sum_{i, j=1}^{n} K\left(t, t_{i}\right) \gamma_{i j n} K\left(t_{j}, \tau\right)
$$

and the function (4) reduces to (10).

Notice that $K_{n}$ coincides with $K$ on $S_{n} \times T$ and $T \times S_{n}$, and consequently, $\hat{f}_{n}=f$ on $S_{n}$. According to the second part of Theorem 1, $K_{n}(t, \cdot)$ converges to $K(t, \cdot)$ in norm and everywhere, and the first part of the proof of the same theorem shows that the sequence (10) converges to $f$ in norm and everywhere.

So, it appears that $\hat{f}_{n}$ gives an approximation of $f$ in norm and everywhere in terms of the values taken by $f$ on the finite subset $S_{n}$ of $S$.

COROLlaRY. The scalar product of any pair of functions $f, g \in \mathfrak{E}_{K}$ is given by 


$$
\langle f, g\rangle=\lim _{n \rightarrow \infty} \sum_{i, j=1}^{n} f\left(t_{i}\right) \gamma_{i j n} \bar{g}\left(t_{j}\right) \text {. }
$$

Consequently, the norm of any function $f \in \mathfrak{F}_{K}$ is given by

$$
\|f\|^{2}=\lim _{n \rightarrow \infty} \sum_{i, j=1}^{n} f\left(t_{i}\right) \gamma_{i j n} \bar{f}\left(t_{j}\right) .
$$

THEOREM $3 .^{1}$ Let $f$ be an arbitrary function defined on $T$, such that

$$
\lim _{n \rightarrow \infty} \sum_{i, j=1}^{n} f\left(t_{i}\right) \gamma_{i j n} \bar{f}\left(t_{j}\right)<\infty, t_{i}, t_{j} \in S, \forall i, j \in N .
$$

Then the sequence of functions defined by

$$
f_{n}(\cdot)=\sum_{i, j=1}^{n} f\left(t_{i}\right) \gamma_{i j n} K\left(t_{j}, \cdot\right)
$$

is a Cauchy sequence in $\mathfrak{S}_{K}$, whose limit $f^{\prime}$ coincides with $f$ on $S$.

Proof. The relation

$$
\left\|f_{m}-f_{n}\right\|^{2}=\left\|f_{m}\right\|^{2}-\left\|f_{n}\right\|^{2}, \quad m>n
$$

holds for the sequence (15), with

$$
\left\|f_{n}\right\|^{2}=\sum_{i, j=1}^{n} f\left(t_{i}\right) \gamma_{i j n} \bar{f}\left(t_{j}\right)
$$

It is then seen that $\left\|f_{n}\right\|^{2}$ is a nondecreasing sequence converging to (14), so that $\left\{f_{n}\right\}$ is a Cauchy sequence in $\mathfrak{C}_{K}$. Let $f^{\prime}$ be its limit. Since $f^{\prime} \in \mathfrak{S}_{K}$, according to Theorem 2, the sequence

$$
\hat{f}_{n}^{\prime}\left(t_{i}\right)=\sum_{i, j=1}^{n} f^{\prime}\left(t_{i}\right) \gamma_{i j n} K\left(t_{j}, \cdot\right)
$$

is also a Cauchy sequence converging to $f^{\prime}$ and therefore $\left\{f_{n}-\hat{f}_{n}^{\prime}\right\}$ converges to the null function in $\mathfrak{L}_{K}$. Since the relation

$$
\begin{gathered}
\left\|f_{m}-\hat{f}_{m}^{\prime}\right\|^{2} \\
\left\|\left(f_{m}-\hat{f}_{m}^{\prime}\right)-\left(f_{n}-\hat{f}_{n}^{\prime}\right)\right\|^{2}=\left\|f_{m}-\hat{f}_{m}^{\prime}\right\|^{2}-\left\|f_{n}-\hat{f}_{n}^{\prime}\right\|^{2}, \quad m>n
\end{gathered}
$$

holds, one has

$$
0 \leqq\left\|f_{n}-\hat{f}_{n}^{\prime}\right\| \leqq \lim _{m \rightarrow \infty}\left\|f_{m}-\hat{f}_{m}^{\prime}\right\|=0
$$

so that $\forall n \in N,\left\|f_{n}-\hat{f}_{n}^{\prime}\right\|=0$. Consequently $\forall t \in T$ and $\forall n \in N, f_{n}(t)=$ $\hat{f}_{n}^{\prime}(t)$. In particular $\forall i \leqq n, f\left(t_{i}\right)=f_{n}\left(t_{i}\right)=\hat{f}_{n}^{\prime}\left(t_{i}\right)=f^{\prime}\left(t_{i}\right)$. Thus, $f(t)=$ $f^{\prime}(t)$, all $t \in S$.

1 This extension was suggested to the author by Professor H. L. Royden. 
It follows from the last theorem that the set $\mathscr{F}$ of all functions satisfying the condition (14) is a Hilbert space in which the scalar product of $f$ by $g$ is given by

$$
\lim _{n \rightarrow \infty} \sum_{i, j=1}^{n} f\left(t_{i}\right) \gamma_{i j n} \bar{g}\left(t_{j}\right), \quad t_{i}, t_{j} \in S, \forall i, j \in N .
$$

In this space all the functions coinciding on $S$ belong to the same equivalence class defined by the relation

$$
f \sim g \Leftrightarrow \lim _{n \rightarrow \infty} \sum_{i, j=1}^{n}\left[f\left(t_{i}\right)-g\left(t_{i}\right)\right] \gamma_{i j n}\left[\bar{f}\left(t_{j}\right)-\bar{g}\left(t_{j}\right)\right]=0 .
$$

In particular, the function $f \in \mathscr{F}$ and the function $f^{\prime} \in \mathfrak{S}_{K}$ corresponding to $f$ as the limit of the sequence (15) are equivalent.

3. Hilbert Space generated by a second order random process. Let $(\Omega, \Sigma, P)$ be a probability space, where $\Omega$ is a sample space, $\Sigma$ is the $\sigma$-algebra generated by a class of subsets of $\Omega$ and $P$ a probability measure defined on $\Sigma$. Let $\left\{X_{t}, t \in T\right\}$ be a class of complex valued random variables defined on $\Omega$ and measurable with respect to $\Sigma$. The symbol $E$ will denote the mathematical expectation with respect to the probability measure $P$. It will be supposed that $\forall t \in T, E\left(X_{t}\right)=0$ and $E\left(\left|X_{t}\right|^{2}\right)<\infty$. The covariance function $E\left(X_{t} \bar{X}_{\tau}\right)$ of thus defined second order stochastic process will be denoted by $K(t, \tau)$.

Let $L_{X}$ be the linear set of all finite linear combinations

$$
\sum_{i} \lambda_{i} X_{t_{i}}, t_{i} \in T, \lambda_{i} \in C .
$$

A scalar product on $L_{X}$ can be defined for any ordered pair of elements

$$
Y=\sum_{i} \lambda_{i} X_{t_{i}}, Z=\sum_{j} \mu_{j} X_{t_{j}}
$$

by the bilinear form

$$
E(Y \bar{Z})=\sum_{i, j} \lambda_{i} \bar{\mu}_{j} K\left(t_{i}, t_{j}\right)
$$

which induces, for any element $Y \in L_{X}$, a norm whose square is defined by

$$
E\left(|Y|^{2}\right)=\sum_{i, j} \lambda_{i} \bar{\lambda}_{j} K\left(t_{i}, t_{j}\right)
$$

The Hilbert space which is the closure of $L_{X}$ in the topology induced by this norm will be denoted by $\mathfrak{S}_{X}$ and will be said to be generated by the process $\left\{X_{t}, t \in T\right\}$.

The theorem of Moore says that there exists a uniquely defined Hilbert space $\mathfrak{S}_{K}$ of functions on $T$, admitting $K$ as its reproducing 
kernel. The construction of $\mathfrak{S}_{X}$ and of $\mathfrak{S}_{K}$ shows that these two spaces are isomorphic if $K$ is the covariance function of $\left\{X_{t}, t \in T\right\}$. Under this isomorphism, the random variable $X_{t}$ corresponds obviously to $K(t, \cdot)$. Consequently, the two spaces are simultaneously separable and if $\left\{K\left(t_{i}, \cdot\right), t_{i} \in S\right\}$ is a basis for $\mathfrak{S}_{K}$ in the sense given in Theorem 1 , then $\left\{X_{t_{i}}, t_{i} \in S\right\}$ is a basis for $\mathfrak{S}_{x}$.

Given an element $Z$ in $\mathfrak{S}_{X}$, the element $f_{Z}$ in $\mathfrak{Q}_{K}$ corresponding to $Z$ is given by

$$
f_{Z}(t)=\left\langle f_{Z}, K(t, \cdot)\right\rangle=E\left(Z \bar{X}_{t}\right) .
$$

For separable $\mathfrak{S}_{K}$ (or equivalently $\mathfrak{S}_{X}$ ) the following theorem gives a representation of the element of $\mathfrak{S}_{X}$ corresponding to any given function $f$ in $\mathfrak{S}_{K}$. The symbols have exactly the same meaning as in the two preceding theorems.

THEOREM 4. For any function $f \in \mathfrak{S}_{K}$, the stochastic element $X(f) \in \mathfrak{S}_{X}$ corresponding to $f$ under the isomorphism between $\mathfrak{S}_{K}$ and $\mathfrak{S}_{X}$, is given by the limit in the quadratic mean of

$$
X\left(\hat{f}_{n}\right)=\sum_{i, j=1}^{n} f\left(t_{i}\right) \gamma_{i j n} X_{t_{j}}
$$

as $n \rightarrow \infty$.

Proof. By replacing $X\left(t_{j}\right)$ by $K\left(t_{j}, \cdot\right)$ in (17), it is seen that $X\left(\hat{f}_{n}\right)$ is the element of $\mathfrak{E}_{X}$ corresponding to $(10)$. Since $\left\{\hat{f}_{n}\right\}$ is a Cauchy sequence in $\mathfrak{S}_{K}$ converging to $f$. Then $\left\{X\left(\hat{f}_{n}\right)\right\}$ is a Cauchy sequence converging to $X(f)$.

In view of the analogy between (12) and (17), the element $X(f)$ can be represented, following Parzen, as $\left\langle f(\cdot), \bar{X}_{(\cdot)}\right\rangle$. But this is not really a scalar product because, almost surely, $X_{(\cdot)}$ does not belong to $\mathfrak{S}_{K}$.

The author would like to thank Professor H. L. Royden, who visited METU in 1966, for helpful discussions.

\section{REFERENCES}

1. N. Aronszajn, Theory of Reproducing Kernels. Trans. Amer. Math. Soc., 68 (1950), 337-404.

2. E. Parzen, Regression Analysis of Continuous Parameter Time Series, Proc. Fourth Berkeley Sym. Prob. Math. Statist., I., University of California Press, (1961), 469489.

3. - Probability Density Functionals and Reproducing Kernel Hilbert Spaces, Time Series Analysis Edit, M. Rosenblatt. J. Wiley, (1963), 155-169.

4. J. Ricatte, Application de la Theorie des Noyaux Reproduisants à des Problèmes 
de Détection et d'Estimation de Signaux, Thèse de Doctorat du Troisième Cycle. Fac. Sc. Paris (1966).

Received November 1, 1966, and in revised form November 27, 1967.

Middle East Technical University

ANKARA, TURKEY 


\section{PACIFIC JOURNAL OF MATHEMATICS}

\section{EDITORS}

\section{H. ROYDEN}

Stanford University

Stanford, California
J. DugundJI

Department of Mathematics

Rice University

Houston, Texas 77001

RICHARD ARENS

University of California

Los Angeles, California 90024

Seattle, Washington 98105

\section{ASSOCIATE EDITORS}
E. F. BeCKENBACH
B. H. NeumanN
F. WOLF
K. YOSIDA

\section{SUPPORTING INSTITUTIONS}

\author{
UNIVERSITY OF BRITISH COLUMBIA \\ CALIFORNIA INSTITUTE OF TECHNOLOGY \\ UNIVERSITY OF CALIFORNIA \\ MONTANA STATE UNIVERSITY \\ UNIVERSITY OF NEVADA \\ NEW MEXICO STATE UNIVERSITY \\ OREGON STATE UNIVERSITY \\ UNIVERSITY OF OREGON \\ OSAKA UNIVERSITY \\ UNIVERSITY OF SOUTHERN CALIFORNIA
}

\author{
STANFORD UNIVERSITY \\ UNIVERSITY OF TOKYO \\ UNIVERSITY OF UTAH \\ WASHINGTON STATE UNIVERSITY \\ UNIVERSITY OF WASHINGTON \\ * * * * \\ AMERICAN MATHEMATICAL SOCIETY \\ CHEVRON RESEARCH CORPORATION \\ TRW SYSTEMS \\ NAVAL WEAPONS CENTER
}

Mathematical papers intended for publication in the Pacific Journal of Mathematics should be in typed form or offset-reproduced, double spaced with large margins. Underline Greek letters in red, German in green, and script in blue. The first paragraph or two must be capable of being used separately as a synopsis of the entire paper. It should not contain references to the bibliography. Manuscripts, in duplicate if possible, may be sent to any one of the four editors. All other communications to the editors should be addressed to the managing editor, Richard Arens, University of California, Los Angeles, California 90024.

Each author of each article receives 50 reprints free of charge; additional copies may be obtained at cost in multiples of 50 .

The Pacific Journal of Mathematics is published monthly. Effective with Volume 16 the price per volume (3 numbers) is $\$ 8.00$; single issues, $\$ 3.00$. Special price for current issues to individual faculty members of supporting institutions and to individual members of the American Mathematical Society: $\$ 4.00$ per volume; single issues $\$ 1.50$. Back numbers are available.

Subscriptions, orders for back numbers, and changes of address should be sent to Pacific Journal of Mathematics, 103 Highland Boulevard, Berkeley 8, California.

Printed at Kokusai Bunken Insatsusha (International Academic Printing Co., Ltd.), 7-17, Fujimi 2-chome, Chiyoda-ku, Tokyo, Japan.

PUBLISHED BY PACIFIC JOURNAL OF MATHEMATICS, A NON-PROFIT CORPORATION

The Supporting Institutions listed above contribute to the cost of publication of this Journal, but they are not owners of publishers and have no responsibility for its content or policies. 


\section{Pacific Journal of Mathematics \\ Vol. 25, No. $2 \quad$ October, 1968}

Martin Aigner, On the tetrahedral graph ..................... 219

Gregory Frank Bachelis, Homomorphisms of annihilator Banach

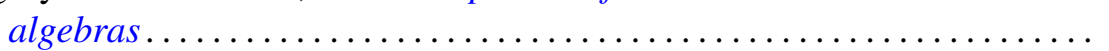

Phillip Alan Griffith, Transitive and fully transitive primary abelian groups.......................................... 249

Benjamin Rigler Halpern, Fixed points for iterates . . . . . . . . . . . . . 255

James Edgar Keesling, Mappings and dimension in general metric spaces ......................................... 277

$\mathrm{Al}$ (Allen Frederick) Kelley, Jr., Invariance for linear systems of ordinary differential equations ................................ 289

Hayri Korezlioglu, Reproducing kernels in separable Hilbert spaces . . . . . 305

Gerson Louis Levin and Wolmer Vasconcelos, Homological dimensions and Macaulay rings ................................. 315

Leo Sario and Mitsuru Nakai, Point norms in the construction of harmonic

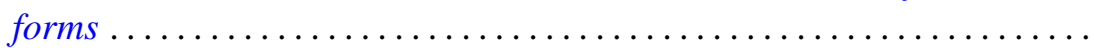

Barbara Osofsky, Noncommutative rings whose cyclic modules have cyclic injective hulls ..................................... 331

Newton Tenney Peck, Extreme points and dimension theory........... 341

Jack Segal, Quasi dimension type. II. Types in 1-dimensional spaces ...... 353

Michael Schilder, Expected values of functionals with respect to the Ito distribution ...

Grigorios Tsagas, A Riemannian space with strictly positive sectional curvature

John Alexander Williamson, Random walks and Riesz kernels . . 